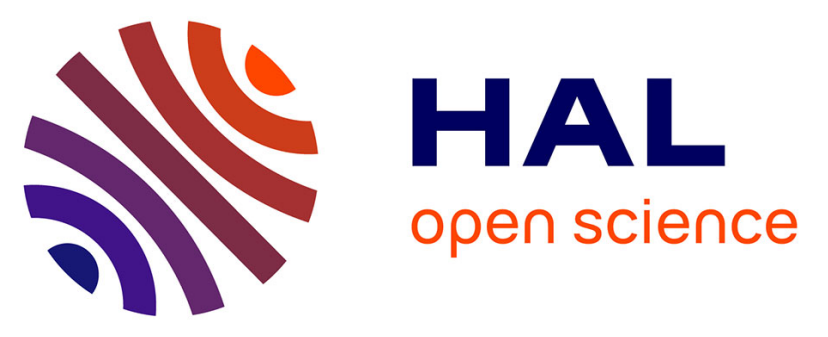

\title{
Elastic anisotropy and single-crystal moduli of solid argon up to 64 GPa from time-domain Brillouin scattering
}

Samuel Raetz, Maju Kuriakose, Philippe Djemia, Sergey Nikitin, Nikolay Chigarev, Vincent Tournat, Alain Bulou, Alexey Lomonosov, Vitali Goussev, Andreas Zerr

\section{To cite this version:}

Samuel Raetz, Maju Kuriakose, Philippe Djemia, Sergey Nikitin, Nikolay Chigarev, et al.. Elastic anisotropy and single-crystal moduli of solid argon up to 64 GPa from time-domain Brillouin scattering. Physical Review B: Condensed Matter and Materials Physics (1998-2015), 2019, 99 (224102), 10.1103/PhysRevB.99.224102 . hal-02316903

\section{HAL Id: hal-02316903 https://hal.science/hal-02316903}

Submitted on 9 Jul 2021

HAL is a multi-disciplinary open access archive for the deposit and dissemination of scientific research documents, whether they are published or not. The documents may come from teaching and research institutions in France or abroad, or from public or private research centers.
L'archive ouverte pluridisciplinaire HAL, est destinée au dépôt et à la diffusion de documents scientifiques de niveau recherche, publiés ou non, émanant des établissements d'enseignement et de recherche français ou étrangers, des laboratoires publics ou privés. 


\title{
Elastic anisotropy and single-crystal moduli of solid argon up to $64 \mathrm{GPa}$ from time-domain Brillouin scattering
}

\author{
Samuel Raetz, ${ }^{1, *}$ Maju Kuriakose, ${ }^{1}$ Philippe Djemia, ${ }^{2}$ Sergey M. Nikitin, ${ }^{1}$ Nikolay Chigarev, ${ }^{1}$ Vincent Tournat,${ }^{1}$ Alain Bulou, ${ }^{3}$ \\ Alexey Lomonosov, ${ }^{4}$ Vitalyi E. Gusev,${ }^{1, \dagger}$ and Andreas Zerr ${ }^{2, \$}$ \\ ${ }^{1}$ Laboratoire d'Acoustique de l'Université du Mans, LAUM - UMR 6613 CNRS, Le Mans Université, Avenue Olivier Messiaen, \\ 72085 LE MANS CEDEX 9, France \\ ${ }^{2}$ Laboratoire des Sciences des Procédés et des Matériaux, LSPM - UPR 3407 CNRS, Université Paris 13, 93430 Villetaneuse, France \\ ${ }^{3}$ Institut des Molécules et Matériaux du Mans, IMMM - UMR 6283 CNRS, Le Mans Université, Avenue Olivier Messiaen, \\ 72085 LE MANS CEDEX 9, France \\ ${ }^{4}$ Prokhorov General Physics Institute, Russian Academy of Sciences, 119991 Moscow, Russian Federation
}

(Received 13 March 2019; revised manuscript received 2 June 2019; published 20 June 2019)

\begin{abstract}
Single-crystal elastic moduli $C_{i j}$, shear modulus $G$, and Zener anisotropic ratio $A$ of solid argon having the face-centered cubic (fcc) structure were determined at high pressures between 12 and $64 \mathrm{GPa}$ using a combination of experimental and theoretical approaches. The experimental data, namely, the maximal and minimal values of the product of the longitudinal sound velocity and refractive index $n \cdot V_{L}$, were obtained from the 3D scanning of elastic inhomogeneities in compressed samples of argon using the technique of time-domain Brillouin scattering. These inhomogeneities, caused by elastic anisotropy of solid argon, were revealed to be about twice as strong as those reported in the earlier experiments using classical Brillouin light scattering (BLS). To derive the $V_{L}$ values, we used the refractive index obtained here from ab initio calculations which also permitted us to rule out any contribution to the amplitude of the observed elastic inhomogeneities of the hexagonal close-packed phase of argon, proposed to coexist with the fcc phase at high pressures. From the measured $C_{i j}(P)$, we derived pressure dependence of shear modulus of the fcc argon $G_{H}(P)$ using the Voigt-Reuss-Hill approximation and found a very good agreement with the earlier $G(P)$ obtained from shear sound velocities in the classical BLS measurements. Our results agree very well with the earlier predictions based on a relatively simple many-body model employing the Buckingham pair potential. Finally, our measurements show a much weaker change of the Cauchy discrepancy $\left(C_{12}-C_{44}-2 P\right)$ of the fcc argon with pressure than reported in all earlier experimental and theoretical works.
\end{abstract}

DOI: 10.1103/PhysRevB.99.224102

\section{INTRODUCTION}

Solid argon, crystallizing at pressures above $1.3 \mathrm{GPa}$ in the face-centered cubic (fcc) structure, is considered as the paradigm of a simple classical solid [1] due to a highly symmetric distribution of electron density in the atoms having completely filled electron shells. For this reason, solid argon is also one of the most intensively investigated objects, also by theory, aiming at understanding the behavior of solids on compression. At first glance, symmetric electronic shells and cubic structure of solid argon imply isotropy of elastic properties of its crystals, which was, however, not confirmed experimentally, especially at elevated pressures. A significant and growing-with-compression contribution of noncentral interaction between the Ar atoms was proposed but still not quantified due to inconsistent experimental observations (see below). Moreover, an experimental report on coexistence of the fcc polymorph with that having the hexagonal close-packed (hcp) structure above $\sim 50 \mathrm{GPa}$ has further complicated the understanding of the compressional

\footnotetext{
*samuel.raetz@univ-lemans.fr

†vitali.goussev@univ-lemans.fr

‡zerr@univ-paris13.fr
}

behavior of this seemingly simple solid [2]. The existing theoretical approaches predicted, however, much higher transition pressures above $220 \mathrm{GPa}$ [3-5] which could be caused by a very small energy difference between the two phases. Also, measurements to 8.2 GPa showed that solid argon (as well as other solidified noble gases) is extremely compressible and produces a hydrostatic environment, as concluded on the basis of the sharpness of its x-ray diffraction (XRD) peaks [6]. Due to the strong change of its lattice constant with pressure, solid argon was suggested and used as an internal pressure standard [7-9].

Elastic anisotropy manifests itself in changing the longitudinal sound velocity $V_{L}$ (as well as the transversal one, $V_{T}$ ) with the direction of the acoustic wave propagation in a single crystal. For solid argon having the fcc structure, compressional waves with the maximal and minimal $V_{L}$ propagate along the crystallographic directions $\langle 111\rangle$ and $\langle 100\rangle$, respectively, designated here as $V_{L\langle 111\rangle}$ and $V_{L\langle 100\rangle}$, e.g., Refs. [10-14]. Their values are given by the expressions $V_{L\langle 111\rangle}=\left(C^{*} / \rho\right)^{1 / 2}=\left[\left(C_{11}+\right.\right.$ $\left.\left.2 C_{12}+4 C_{44}\right) /(3 \rho)\right]^{1 / 2}$ and $V_{L\langle 100\rangle}=\left(C_{11} / \rho\right)^{1 / 2}$, where $C_{i j}$ are single-crystal elastic moduli. Elastic anisotropy can also be quantified, for cubic crystals, through a deviation of the Zener anisotropic ratio $A=2 C_{44} /\left(C_{11}-C_{12}\right)$ from unity. 

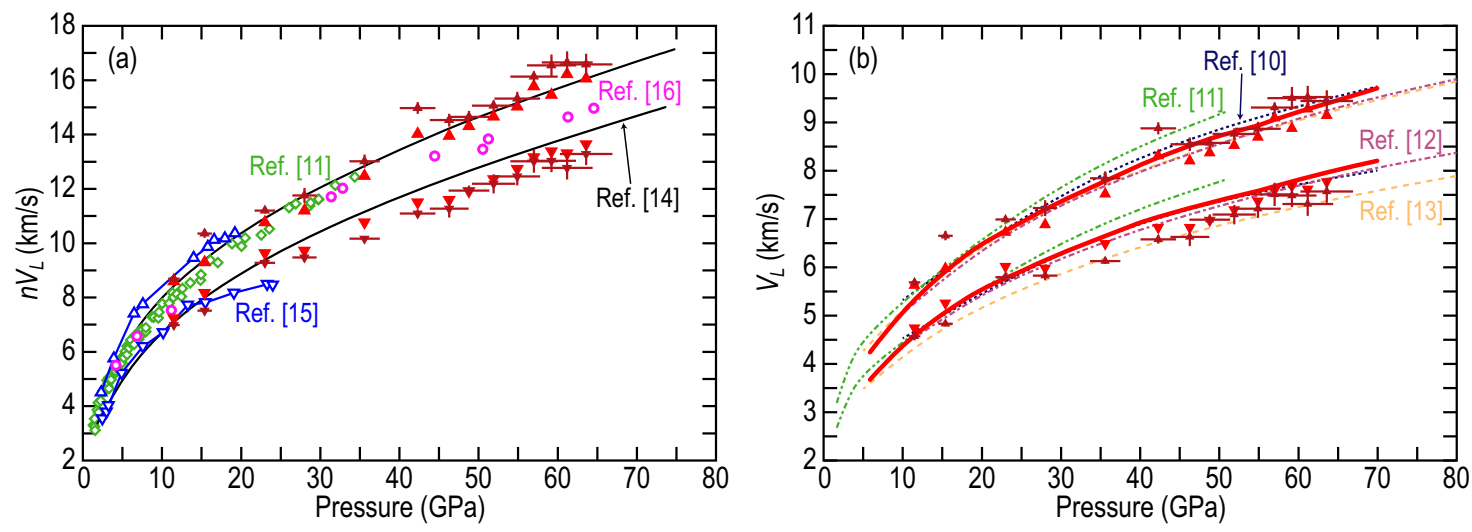

FIG. 1. Experimental and theoretical data on longitudinal sound velocities of solid argon at high pressures. (a) Our results in terms of the product $n \cdot V_{L}$ obtained using the TDBS technique, represented by triangles pointing up and down, correspond to the maximal and minimal values, respectively. The representation in terms of $n \cdot V_{L}$ was used here because it is not influenced by the choice of the refractive index. The smaller (dark red) and bigger (red) solid triangles indicate our results for the narrower and wider temporal window of one and two-and-a-half Brillouin oscillations, respectively. Experimental uncertainties in $n \cdot V_{L \max }$ and $n \cdot V_{L \min }$ are shown for the narrower temporal window only, because for the wider one the uncertainties are smaller than the symbols. The pressure uncertainties are the same for both data sets. Thin solid (black) lines as well as the open (blue) triangles pointing up and down represent $n \cdot V_{L \max }$ and $n \cdot V_{L \min }$, respectively, measured using the classical BLS in Refs. [14,15]. Open (green) diamonds and open (magenta) circles indicate averaged values $n \cdot V_{L \text { av }}$ obtained using the same technique $[11,16]$. To be able to compare results reported in Refs. [14,16], we multiplied their directly measured $V_{L}$ values with our theoretical $n(P)$ shown below in Fig. 5. (b) Our experimental results on $V_{L \max }$ and $V_{L \min }$ compared with the theoretical calculations of $V_{L\langle 111\rangle}$ and $V_{L\langle 100\rangle}$ for the fcc argon as a function of pressure. Our theoretical results are shown by thick solid (red) lines. Broken lines represent results of the earlier theoretical calculations: Dashed (orange) line—Pechenik et al. [13]; dotted (dark blue) line-Aoki and Kurokawa [10]; dash-dotted (dark pink) line-Iitaka and Ebisuzaki [12]; dash-double-dotted (green) line-Grimsditch et al. [11].

$V_{L\langle 111\rangle}$ and $V_{L\langle 100\rangle}$ represent the extremes for experimentally obtained maximal and minimal longitudinal sound velocities, $V_{L \max }$ and $V_{L \min }$, in classical BLS measurements on polycrystalline fcc argon compressed in a diamond anvil cell (DAC). A significant deviation of $n \cdot V_{L \max }$ from $n \cdot V_{L \text { min }}$ (where $n$ is the refractive index) was first recognized in experiments up to $\sim 34 \mathrm{GPa}$ [Fig. 1(a)] [11], where smooth curves embracing the scattering $V_{L}$ values at different pressures, $V_{L \max }(P)$ and $V_{L \min }(P)$, were attributed to $V_{L\langle 111\rangle}(P)$ and $V_{L\langle 100\rangle}(P)$, respectively. From this so-called envelope method, the $C_{i j}(P)$ dependencies for the fcc argon were derived and the Zener ratio $A$ greater than 1.97 at $P \simeq 2 \mathrm{GPa}$ and greater than 1.16 at $P=30 \mathrm{GPa}$ obtained [11]. However, the $C_{i j}(P)$ predicted theoretically in the same work [Fig. 2(a)] showed a much stronger deviation of $V_{L\langle 111\rangle}$ from $V_{L\langle 100\rangle}$ [Fig. 1(b)] and, accordingly, a much higher Zener ratio increasing from $A=2.86$ at $P=1.65 \mathrm{GPa}$ to $A=3.67$ at $P=50$ GPa [Fig. 2(b)]. More important, the experimentally found deviation of the Cauchy discrepancy $\left(C_{12}-C_{44}-2 P\right)$ from zero (Fig. 3) suggested that an accurate description of the high-density cubic argon in terms of pairwise atom interactions is impossible [11].

In subsequent classical BLS measurements on single crystals of the fcc argon at $P \leqslant 4 \mathrm{GPa}$, a Zener ratio of $A=3$ was obtained [14]. The reported gradual decrease on further compression to $A=2$ at $P=70 \mathrm{GPa}$ [Figs. 1(a) and 2(b)] could be an artifact because the samples degraded to polycrystals and the envelope method had to be applied [14]. A much stronger deviation of $V_{L \max }(P)$ from $V_{L \min }(P)$, and thus a stronger elastic anisotropy, was recognized by Chen et al. but the Zener ratio was not quantified [15]. The main reason for inconsistent and lower $A$ values obtained for polycrystalline samples in the classical BLS measurements, when compared with those for single crystals, is the fact that it is challenging to get a close approximation of the actual limiting values $V_{L\langle 111\rangle}(P)$ and $V_{L\langle 100\rangle}(P)$. The envelope method requires an unacceptably large number of measurements of weak BLS spectra from microscopic samples compressed in a DAC. The probability to obtain a BLS peak whose maximum corresponds to the limiting $V_{L}$ value is nearly zero because any of the peaks is a superposition of intensities from a large number of differently oriented crystallites along the sample depth. This was also pointed out and critically discussed by Marquardt et al. [16], who have found from a combination of their classical BLS [Fig. 1(a)] and XRD measurements, that it is very unlikely that their $V_{L \max }(P)$ and $V_{L \min }(P)$ represented the overall "singlecrystal" extreme velocity values, $V_{L\langle 111\rangle}(P)$ and $V_{L\langle 100\rangle}(P)$. As a consequence, they have found a pronounced discrepancy between their shear modulus $G(P)$ and that of Shimizu et al. [14], thus supporting the result of Chen et al. [15]. Another outcome of this work was the conclusion that solid argon is a very soft-pressure medium having a low shear strength of only $0.8 \mathrm{GPa}$ at $P=65 \mathrm{GPa}$, similar to that of solid neon [16]. Thus, earlier statements [17] about a limited applicability of solid argon as a pressure transmitting medium (PTM) were disproved.

Theoretical calculations $[10,12,13]$ also predicted a significant and growing-with-pressure elastic anisotropy of solid argon [Figs. 1(b) and 2(b)], outranging that measured in Ref. [14]. However, the theoretical results were inconsistent and predicted deviating dependencies of the Zener ratio and Cauchy discrepancy on pressure [Figs. 2(b) and 3]. The strongest anisotropy was predicted from a simple embeddedatom-type many-body model where the Buckingham 

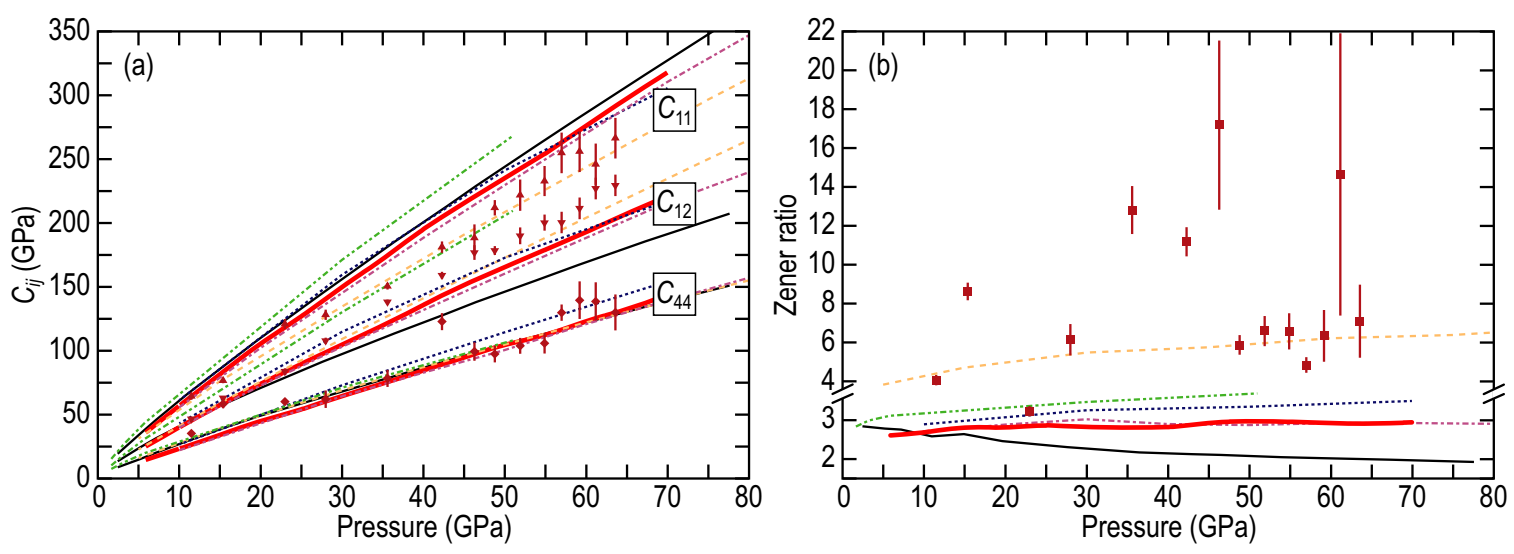

FIG. 2. Pressure dependencies of (a) $C_{i j}$ and of (b) the Zener ratio $A$ of the fcc argon obtained experimentally and theoretically. Except our experimental data, all other theoretical and experimental results are shown in the same style as in Fig. 1. (a) The solid (dark-red) triangles pointing up and down and the rhombuses represent our experimental values of $C_{11}(P), C_{12}(P)$, and $C_{44}(P)$, respectively. Here and below, pressure uncertainties in our experiments (the same as in Fig. 1) are not shown for clarity of the presentation. (b) Our experimental values of the Zener ratio, derived from our experimental $C_{i j}(P)$ shown in (a), are represented by solid (dark-red) squares.

(exp-6) pair potential was applied and the many-body term was derived from a fit to the available-at-that-time experimental data [14] and $a b$ initio calculations [12].

For polycrystalline solids available in microscopic amounts, as is the case for samples at very high pressures, the access to reliable extrema of $V_{L}$ in a single crystal [e.g., $V_{L\langle 111\rangle}(P)$ and $V_{L\langle 100\rangle}(P)$ for a cubic crystal], to $C_{i j}(P)$ and to averaged shear modulus $G(P)$ appears to be possible when $V_{L}$ is measured for each crystallite (typically $<1 \mu \mathrm{m}$ in size). The classical BLS (also called frequency-domain Brillouin spectroscopy) provides a reasonable lateral resolution but not along the laser beam. Accordingly, any collected BLS peak results from the integration of signals coming from all crystallites along the sample depth (i.e., incoming light

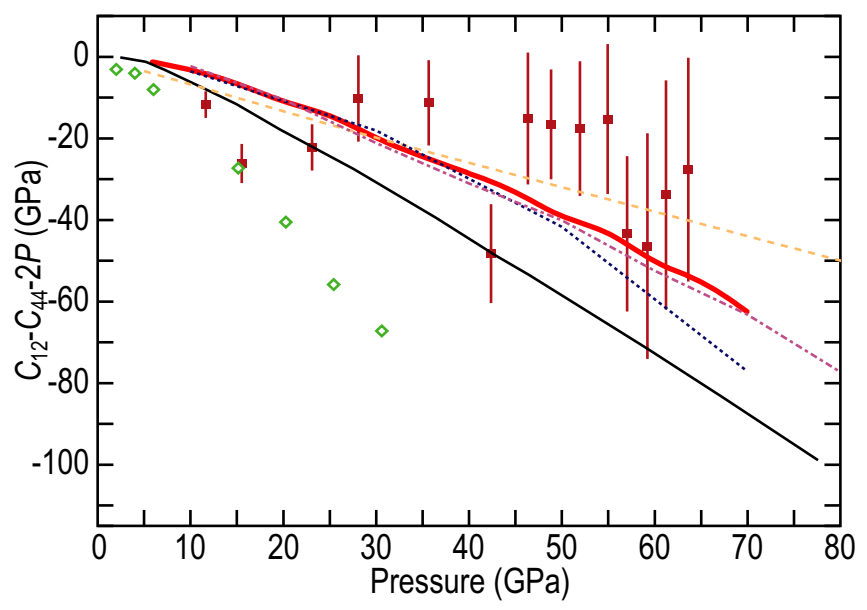

FIG. 3. Theoretical and experimental pressure dependencies of the Cauchy discrepancy $\left(C_{12}-C_{44}-2 P\right)$. Except our experimental data represented by solid (dark-red) squares, all other results are shown in the same style as in Fig. 1. The theoretical result of Ref. [11], predicting that the Cauchy discrepancy approaches zero, is not shown. path). The frequency value to which the BLS peak has maximum gives therefore the "averaged" sound velocity $V_{L \text { av }}$ that approaches, although still is significantly far from, $V_{L\langle 111\rangle}$ for cubic crystals [18]. This is also the case for solid argon, as follows from a comparison of the earlier measured $V_{L \text { av }}(P)$ [11] and $V_{L \max }(P)$ [14,15] in Fig. 1(a). If the examined material is, in addition, plastically anisotropic (e.g., different shear strength for different crystallographic directions), then extended textured regions forming in compressed polycrystalline samples cause BLS peaks having varying shapes and maximum positions. In such case, even averaged elastic moduli cannot be determined reliably, not to mention the estimation of elastic anisotropy from the peak shapes. Here, we did not consider the contribution of photoelastic anisotropy which also can influence the BLS peak shape.

It was shown in our recent work that the technique of time-domain Brillouin scattering (TDBS) [19] permits 3Dscanning of transparent samples with sub- $\mu \mathrm{m}$ axial resolution, in addition to the micrometric lateral resolution [18,20,21]. In the present paper, we applied this capability of the TDBS technique to obtain 3D distributions of elastic inhomogeneities in polycrystalline samples of the fcc argon compressed in a DAC [20] and to pitch the extreme $n \cdot V_{L}$ values closely approaching the $n \cdot V_{L\langle 111\rangle}$ and $n \cdot V_{L\langle 100\rangle}$ up to the highest pressure of our work of $64 \mathrm{GPa}$ (Fig. 1). An important part of the work was a theoretical calculation of refractive index of the fcc argon (at the wavelength of the probe beam, $\lambda_{L}=808 \mathrm{~nm}$ ) as a function of pressure $n(P)$ needed to derive $V_{L}$ values from the Brillouin frequencies $f_{B}$ (or from the product $n \cdot V_{L}$ ) we obtained directly from TDBS signals [18]. To our knowledge, only one work, published more than 30 years ago, dealt with prediction of $n(P)$ for cubic argon [11]. The obtained $n(P)$ was reported to be consistent with the earlier measurements of the same group of authors. However, those measurements were limited to a maximal pressure of $\sim 16 \mathrm{GPa}$ and the accuracy was rather moderate [22]. Moreover, that experimental $n(P)$ deviated from the recent but similarly imprecise data up to $\sim 27 \mathrm{GPa}$ [15] (Fig. 5). Our analysis showed that a relatively small difference in $n(P)$ of $\sim 10 \%$ leads to 
doubling the Zener ratio $A$ when applied to our experimental dependencies $n(P) \cdot V_{L \max }(P)$ and $n(P) \cdot V_{L \min }(P)$. Accordingly, we performed independent calculations of $n(P)$ using a stateof-the-art $a b$ initio approach to determine $V_{L\langle 111\rangle}(P)$ and $V_{L\langle 100\rangle}(P)$ of the fcc argon from our TDBS data. The applied method insured lower numerical errors in the refractive index when compared with the above experimental results, of the order of $1 \%$ or less. This accuracy required particular attention in the optimization of $k$-mesh of the Brillouin zone, of energy cutoff for the plane-wave expansion, and of acceptable duration of the calculations. Apparently, intrinsic uncertainty due to the underlying theory, e.g., the chosen exchange-correlation energy functional, cannot be verified otherwise than by comparison with experiments.

Thereafter, applying the earlier measured bulk modulus $B(P)$, we derived $C_{i j}(P)$, averaged polycrystalline shear modulus $G_{H}(P)$, and pressure dependence of the Cauchy discrepancy. Furthermore, we extended our calculations to the hcp phase of solid argon with the aim to verify that this phase exhibits a weaker elastic anisotropy in comparison with the fcc phase. If not the case, the attributing of the dependencies $n(P) \cdot V_{L \max }(P)$ and $n(P) \cdot V_{L \min }(P)$ from our TDBS measurements to $n(P) \cdot V_{L\langle 111\rangle}(P)$ and $n(P) \cdot V_{L\langle 100\rangle}(P)$ of the fcc argon, respectively, would be inappropriate.

\section{METHODS}

A comprehensive description of the experimental techniques used in this paper, together with a discussion of potential limitations of the TDBS technique due to the intrinsic state of compressed samples and estimations of the uncertainties, can be found elsewhere [18,20,21]. Only a short summary of the experimental procedure and of the particularities specific for the examined material are considered here. Samples of solid argon were compressed between 11 and 64 GPa using a piston-cylinder type DAC [23] with beveled anvils having culets of $350 \mu \mathrm{m}$ in diameter. The samples were obtained by sealing argon, compressed to $3000 \mathrm{~atm}$ in a gas-loading apparatus, in a hole of 100-150 $\mu \mathrm{m}$ in diameter drilled in a preindented stainless steel gasket. The sample volume having initial thickness of typically less than $40 \mu \mathrm{m}$ contained a $\sim 2 \mu$ m-thick and $\sim 100 \mu$ m-diameter iron disk sticking to the culet of the anvil mounted on the piston. This disk served as an optoacoustic generator of coherent acoustic pulses launched into-and propagating through - the sample. A few ruby grains, distributed around the optoacoustic generator, were used to determine the pressure in the sample volume from the shift of the $R 1$ fluorescence line calibrated earlier via a compression of metals with known equations of state (EOS) in the argon pressure medium [24].

The 3D distributions of $V_{L}$ in samples of solid argon compressed in the DAC were measured using the TDBS setup described in detail elsewhere [18,20,21]. In this setup, coherent phonons were generated and their propagation was monitored using a pulsed Ti:Sapphire laser (Spectra-Physics, $2 \mathrm{~W}, 808 \mathrm{~nm}, 2.7 \mathrm{ps}$ pulse width) whose radiation was split into two beams and the photon frequency of one of them was doubled (404 nm, 1.9 ps pulse width). The latter laser beam (pump) was focused on the optically absorbing optoacoustic generator (a thin iron disk) which launched, due to subsequent

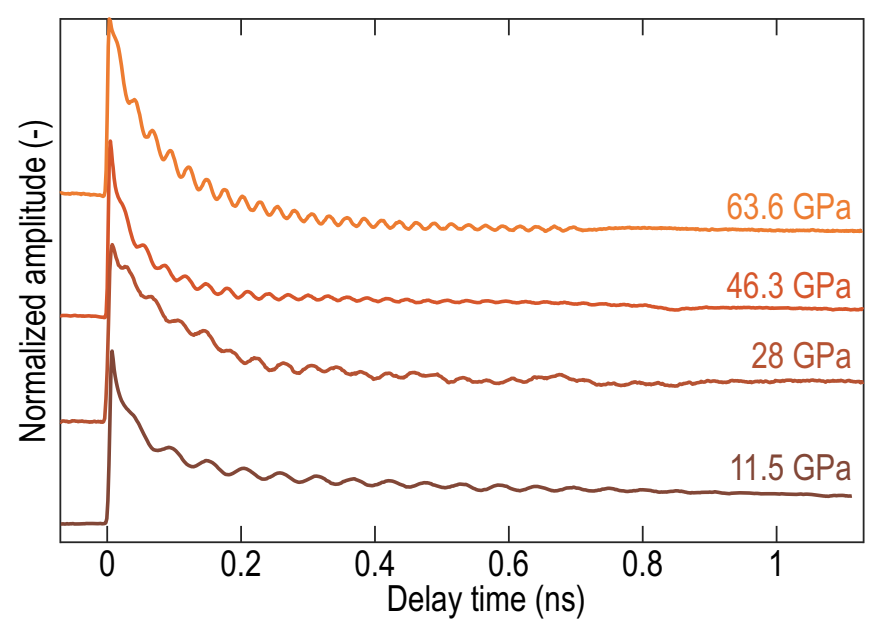

FIG. 4. Raw transient reflectivity signals $S(t)$ for pressures of (bottom to top): $11.5 \mathrm{GPa}, 28 \mathrm{GPa}, 46.3 \mathrm{GPa}$, and 63.6 GPa.

transient heating and expansion, ultrashort acoustic pulses into the sample. Time-delayed laser pulses of the second beam (probe) with the fundamental wavelength permitted us to follow propagation of the acoustic pulses through the solid argon, which is transparent. Optical paths of the pump and probe beams were normal to the diamond anvil culets and thus to the sample surface. Interference of reflections of the probe beam from different stationary surfaces/interfaces in the DAC and from the moving photo-generated acoustic pulse resulted in a signal, recorded using a photodetector, containing a quasiharmonically oscillating component $S(t)$, where $t$ is the delay time between the pump and the probe laser pulses. Examples of raw transient reflectivity signals $S(t)$ are shown in Fig. 4 for pressures of $11.5 \mathrm{GPa}, 28 \mathrm{GPa}, 46.3 \mathrm{GPa}$, and 63.6 GPa. The mean frequency, and accordingly the average longitudinal sound velocity, is evidently increasing (the period of the Brillouin oscillation is decreasing) with pressure.

At each instant of time $t$, the frequency $f(t)$ of $S(t)$ is related to the product $n \cdot V_{L}$ along the sample axis at the position where the laser-generated picosecond acoustic pulse is located during its propagation through the sample. The lateral resolution of our TDBS measurement of $2.7 \mu \mathrm{m}$ was defined by focusing the pump and probe beams [20]. The axial resolution of less than $0.1 \mu \mathrm{m}$ was limited by the width of the acoustic pulse [18,21]. Frequency of the collected TDBS signal (and its variations with lateral coordinate and depth due to elastic anisotropy of the sample material) is proportional to the longitudinal sound velocity (varying with depth/propagation time), according to the classical formula for normal incidence of the probe beam: $V_{L}(P)=\left(f_{B} \lambda_{L}\right) /[2 n(P)]$ where $f_{B}=f(t)$ is the (delay time-dependent) frequency of the Brillouin oscillations, $\lambda_{L}$ the wavelength of the probe laser beam $\left(\lambda_{L}=808 \mathrm{~nm}\right)$, and $n(P)$ the refractive index of the sample material. Variations of $n \cdot V_{L}$ along the sample axis were tracked with the resolution defined by the chosen temporal window size equal to the duration of $\sim 1$ or $\sim 2.5$ Brillouin oscillations. The wider window permitted us to evaluate the effect of averaging over a larger depth range on the values of $n \cdot V_{L \max }$ and $n \cdot V_{L \min }$. At the lowest pressure of $11.4 \mathrm{GPa}$, these two windows corresponded to the depth resolution 
of $\sim 0.27 \mu \mathrm{m}$ and $\sim 0.68 \mu \mathrm{m}$, respectively. At the highest pressure of the work of $63.6 \mathrm{GPa}$, the depth resolution was $\sim 0.25 \mu \mathrm{m}$ and $\sim 0.62 \mu \mathrm{m}$, respectively. Specific for solid argon was a strong damping of the TDBS signals, independent of pressure. This limited the sample depth accessible for measurements to less than $6 \mu \mathrm{m}$ at lower pressures [20] and less than $8 \mu \mathrm{m}$ at $P=63.6 \mathrm{GPa}$. For this reason, lateral scanning of the $n \cdot V_{L}$ distribution was performed over distances of $60-90 \mu \mathrm{m}$, comparable with the diameter of the optoacoustic generator, to have, after the signal treatment, at least 2000 independent and reliable measurements of $n \cdot V_{L}$ at each pressure point. This procedure permitted a consistent application of the envelope method, where the $n \cdot V_{L \max }$ and $n \cdot V_{L \min }$ values from all our TDBS signals collected at a chosen pressure could be attributed to the maximal and minimal possible values in a single crystal of solid argon, with a higher degree of confidence than in the case of classical BLS measurements. If solid argon maintains the fcc structure in the entire pressure range of our measurements, then our experimental dependencies $n(P) \cdot V_{L \max }(P)$ and $n(P) \cdot V_{L \min }(P)$ will correspond to $n(P) \cdot V_{L\langle 111\rangle}(P)$ and $n(P) \cdot V_{L\langle 100\rangle}(P)$ in a single crystal, respectively.

To obtain reliable axially resolved values of $n \cdot V_{L}$, we applied the same demanding time-frequency analysis of the raw TDBS signals $S(t)$ as described in detail in Ref. [18]: $S(t)$ was initially filtered to remove the nonoscillating background caused by a transient heating of the sample and then transformed to the amplitude-variation-free analytical signal $\exp [j \phi(t)]$. The best fit to the latter signal by the complex harmonic function applying the synchronous detection technique provided the TDBS signal frequency $f_{B}$ at any selected time instant (central time of the moving temporal window). The time instants with poor signal-to-noise ratio (SNR) and/or with a large normalized root-mean-square error (NRMSE) were excluded from the consideration. In this paper, we considered only the time instants where SNR exceeded $10 \mathrm{~dB}$ and NRMSE was below 5\%. More details on the determination of experimental uncertainties in $f_{B}$ and, accordingly, $n \cdot V_{L}$ can be found in our earlier paper [18].

We performed $a b$ initio calculations to obtain $C_{i j}(P)$ and $n(P)$ of the two phases of solid argon having fcc (space group $F m \overline{3} m$ ) and hcp (space group $P 6 \overline{3} / m m c$ ) structures. The calculations were based on the density functional theory (DFT) as implemented in the VIENNA AB-INITIO SimUlation PACKAGE (VASP) $[25,26]$. They were accomplished with tools and functions interfaced to VASP $[27,28]$ within the MATERIAL EXPLORATION AND DESIGN ANALYSIS (MEDEA) software [29], which handles automatic executions of task sequences. The DFT calculations of the geometry optimization as well as of the elastic and optical properties at high hydrostatic pressures were performed using the hybrid functional HSE06 [30-33], as implemented in VASP. It is well known that pure functionals severely underestimate band gaps and, accordingly, predict incorrect optical properties such as refractive index. In contrast, it was shown in the literature for multiple insulating systems (as argon in our case) that calculations using the HSE06 functional are much less demanding with respect to the density of the overall $k$ sampling of a Brillouin zone and that the calculated band gaps match well the experimental ones, better than 10\% [34].
Interaction of the eight valence electrons of $\mathrm{Ar}$ $\left([\mathrm{Ne}] 3 \mathrm{~s}^{2} 3 \mathrm{p}^{6}\right)$ with cations were described by the projector augmented wave method [35] with a plane-wave energy cutoff of $346 \mathrm{eV}$. The energy convergence criterion for electronic self-consistency was set to $3 \times 10^{-6} \mathrm{eV} /$ atom using the damped molecular dynamics algorithm and reciprocal space projection operators. The Monkhorst-Pack scheme [36] was used to construct the $4 \times 4 \times 4 k$-mesh of the Brillouin zone in the case of the fcc structure and the $6 \times 6 \times 3 k$-mesh in the case of the hcp structure for the self-consistent and nonlocal exchange calculations. Two-times-denser $k$-meshes were used for the density of states and optical spectra calculations, namely, $8 \times 8 \times 8$ and $12 \times 12 \times 6$ for the fccand hcp structures, respectively. The $k$-meshes were forced to be centered on the $\Gamma$ point and a Gaussian smearing with a width of $0.2 \mathrm{eV}$ was employed for the relaxation of the crystal structures. The number of bands (NBANDS parameter) was set to 30 . The frequency-dependent optical properties, in particular, the refractive index $n(P)$ at $2.33 \mathrm{eV}$ (corresponding to the wavelength of $532 \mathrm{~nm}$ ) and at $1.55 \mathrm{eV}$ (corresponding to the wavelength of $808 \mathrm{~nm}$ ), were calculated using the TAG parameter LOPTICS=.TRUE. in the VASP software. The complex shift used to smooth the real part of the dielectric function was set to 0.1. A detailed description of the theoretical approach can be found elsewhere [37].

To examine numerical errors by the computations, we performed more demanding calculations, when compared with the above-described optimal ones. In particular, at pressures of $20 \mathrm{GPa}$ and $60 \mathrm{GPa}$, we used for the fcc phase a higher energy cutoff of $1000 \mathrm{eV}$ and much finer $k$-meshes of $13 \times 13 \times 13$ and $15 \times 15 \times 15$, respectively. Again, the meshes were doubled for the calculation of the optical properties. The higher energy cutoff of $700 \mathrm{eV}$ and the $k$-mesh of $13 \times 13 \times 7$ was tested by calculation of properties of the hcp phase at $20 \mathrm{GPa}$. These tests showed that the total energies obtained for the different meshes remained the same within a range of $10 \mathrm{meV}$. In the case of the fcc phase, we obtained minimal deviations of less than $1 \%$ (when compared with those from the optimal calculations) for $C_{i j}(P)$ and bulk modulus $B(P)$, of much less than $1 \%$ for the refractive index $n(P)$, and $<0.2 \%$ for the lattice parameter. All physical values were fully converged with respect to the $k$ grids and energy cutoffs. Accordingly, an overall relative error of $2 \%$ must be considered for the theoretical product $n(P) \cdot V_{L}(P)$.

\section{RESULTS AND DISCUSSIONS}

Prior to the treatment of the TDBS signals collected for solid argon at pressures between $11.5 \mathrm{GPa}$ and $63.6 \mathrm{GPa}$, we calculated the pressure dependence of the refractive index $n(P)$ of the fcc argon at the wavelength of the probe laser beam (Fig. 5). Our theoretical $n(P)$ was found to agree with both earlier reported experimental data $[15,22]$ which overlap due to the significant experimental uncertainties (Fig. 5). Our theoretical $n(P)$ was found to be higher than that calculated in 1986 using an approach accessible at that time [11]. At the highest pressure of our work, the difference between that earlier result and our prediction reached $\sim 7 \%$. As mentioned above, the HSE06 functional was shown to reproduce with a good accuracy electronic and optical properties, such 


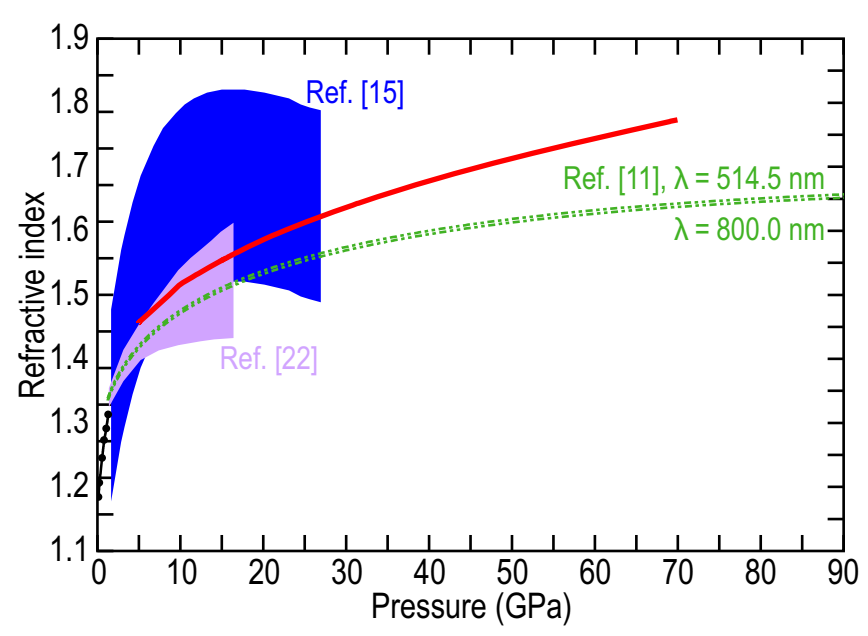

FIG. 5. Experimental and theoretical refractive index of solid argon as a function of pressure. The experimental results of Chen et al. [15] and Grimsditch et al. [22] are shown as solid dark (blue) and light (violet) bands, respectively, embracing the scattering data with error bars. The thick solid (red) line represents our calculated $n(P)$ while the dash-double-dotted lines represent the earlier predicted $n(P)$ for the two wavelengths [11].

as density of states or band gap, for many semiconductors and insulators [34] and, accordingly, variation of the permittivity function around optical transitions. Thus, this functional promises a more reliable calculation of refractive index of an insulating solid at high pressures when compared with other functionals or simple generalized gradient approximation and local density approximation. The belowdescribed very good agreement of our experimental shear modulus $G_{H}(P)$ with that measured independently using the classical BLS, $G(P)$, should be considered as strong support of our calculational approach. For comparison, if we had used $n(P)$ predicted in Ref. [11] then, at the highest pressure of our work, our experimental shear modulus $G_{H}(P)$ would have been $\sim 1.6$ times higher than $G(P)$ from the classical BLS measurements [16].

To verify that our theoretical approach provides correct ground-state properties, we calculated $\operatorname{EOS} \rho(P)$ and bulk modulus $B(P)$ of the fcc argon and compared them with earlier theoretical and experimental results. It should be mentioned here that all experimental data agree within the experimental uncertainties $[2,8,16]$ and all theoretical EOS [10-13], including our $\rho(P)$, do not deviate from the experimental ones by more than $4 \%$. However, the earlier predicted $B(P)$ deviated from the experimental data more strongly than our theoretical $B(P)$ (Fig. 6). We considered the good agreement of our theoretical $\rho(P)$ and $B(P)$ (Fig. 6) as a confirmation of the reliability of our ground-state calculations.

Results of our TDBS measurements on solid argon compressed between $P=11.5 \mathrm{GPa}$ and $P=63.6 \mathrm{GPa}$ are shown in Fig. 1 in two manners: Figure 1(a) presents the raw data, namely the products $n(P) \cdot V_{L \max }(P)$ and $n(P) \cdot V_{L \min }(P)$ which are not influenced by the choice of the refractive index $n(P)$ and, accordingly, of crystal structure of solid argon. In Fig. 1(b), we compare our experimental $V_{L \max }(P)$ and $V_{L \min }(P)$ derived from the data shown in Fig. 1(a) using our

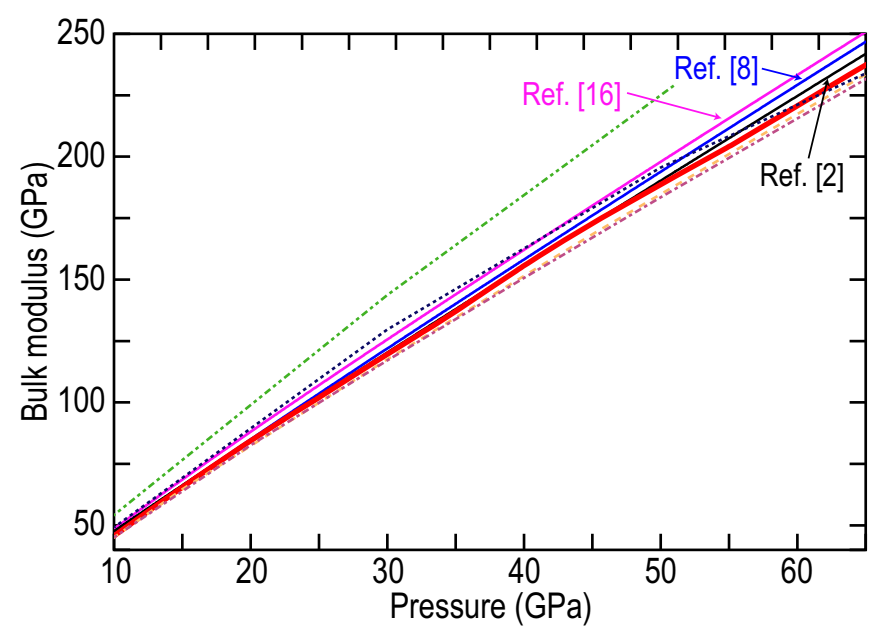

FIG. 6. Comparison of the earlier experimental (thin solid lines) and theoretical (broken lines) $B(P)$ with our calculation (thick solid (red) line). The experimental values were obtained by Richet et al. [8] (blue line), Errandonea et al. [2] (black line), and Marquardt et al. [16] (magenta line). The earlier theoretical results are shown in the same style as in Fig. 1.

theoretical $n(P)$ calculated for the fcc argon (Fig. 5). Our experimental Brillouin frequencies $f_{B}$ (and, accordingly, $n \cdot V_{L}$ values) were extracted from the TDBS signals for two temporal windows of $\sim 1$ or $\sim 2.5$ Brillouin oscillations. The wider temporal window gave a smaller deviation of $n(P) \cdot V_{L \max }(P)$ from $n(P) \cdot V_{L \min }(P)$, which suggests that the characteristic size of elastic inhomogeneities in our argon sample along its depth is well below $0.6 \mu \mathrm{m}$. The deviation of $n(P) \cdot V_{L \max }(P)$ from $n(P) \cdot V_{L \min }(P)$ for the wider temporal window was found to be similar to that obtained earlier in classical BLS measurements at pressures below $35 \mathrm{GPa}$ [14] [Fig. 1(a)] but became stronger at higher pressures. The deviation of $n(P) \cdot V_{L \max }(P)$ from $n(P) \cdot V_{L \min }(P)$ for the narrower temporal window was measured to be much stronger than that in the earlier BLS measurements of Shimizu et al. [14]. Interestingly, our data agree reasonably well with $n(P) \cdot V_{L \max }(P)$ and $n(P) \cdot V_{L \min }(P)$ obtained by Chen et al. at $P<25 \mathrm{GPa}$ [15] who used the classical BLS as well [Fig. 1(a)]. The dependence of averaged values $n(P) \cdot V_{L \text { av }}(P)$ measured for homogeneous polycrystalline samples in Ref. [11] approaches our $n(P) \cdot V_{L \max }(P)$ as well as that reported earlier in Refs. [14,15]. This fact we explained in our previous work on cubic $\mathrm{H}_{2} \mathrm{O}$ ice by a strongly asymmetric shape of the peaks in classical BLS measurements [18].

In the following data treatment, we first applied our $n(P)$ calculated for the fcc argon to derive the $V_{L \max }(P)$ and $V_{L \min }(P)$. Then we used only those obtained for the narrower temporal window because they approached closely the $V_{L}$ extreme values in a single crystal of the fcc argon. In the case of cubic argon, $V_{L \max }(P)$ and $V_{L \min }(P)$ correspond, according to the earlier and to our theoretical calculations, to $V_{L\langle 111\rangle}(P)$ and $V_{L\langle 100\rangle}(P)$, respectively. To derive $C_{i j}(P)$, we used an additional independent experimental value, namely, $\rho(P)$. We considered three $\rho(P)$ measured using the XRD technique $[2,8,16]$ which are very similar (see above) with the maximal difference in $B(P)$ below $4 \%$ (Fig. 6). We selected the interme- 

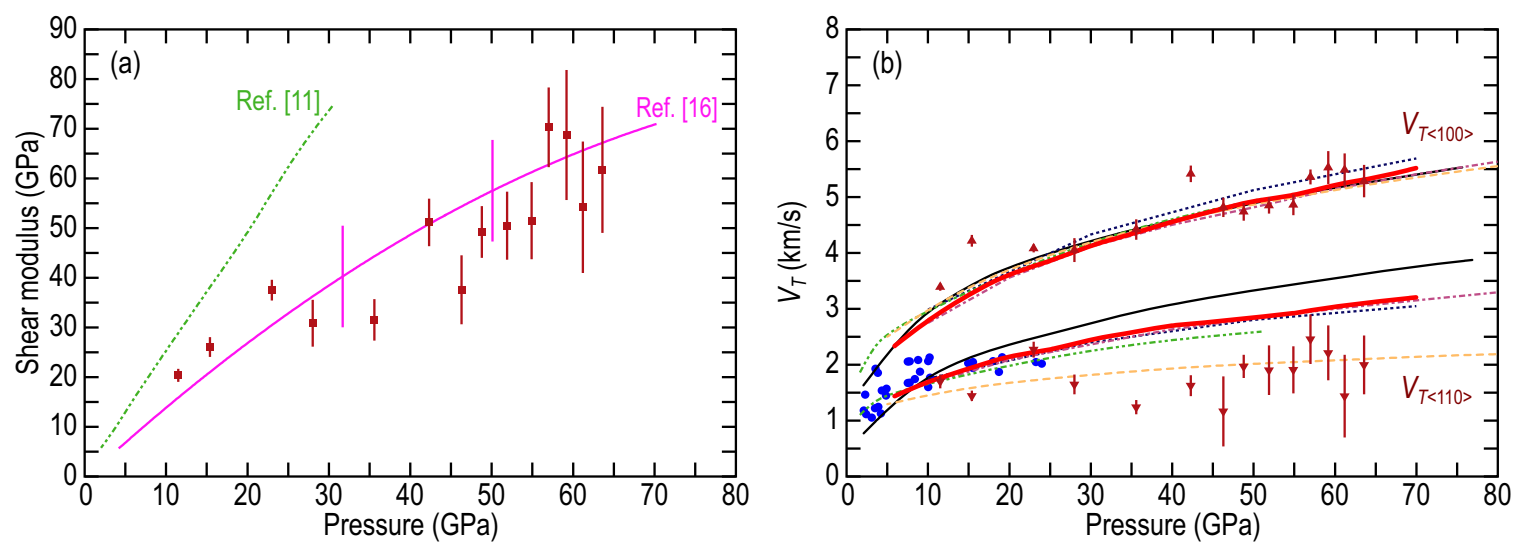

FIG. 7. (a) Pressure dependence of the shear modulus $G_{H}$ of polycrystalline fcc argon derived from our experimental $C_{i j}(P)$ using the VoigtReuss-Hill approximation (solid dark-red squares) in comparison with the earlier reported $G(P)$ obtained in the classical BLS experiments by Marquardt et al. [16] from the averaged $V_{T \text { av }}(P)$ (solid magenta line). $G_{H}(P)$ derived from the earliest experimental $C_{i j}(P)$ reported in Ref. [11] are represented by the (green) dash-double-dotted line. Vertical bars show experimental uncertainties for our experimental data-set and for that of Marquardt et al. [16]. (b) $V_{T\langle 100\rangle}(P)$ (solid dark-red triangles pointing up) and $V_{T\langle 110\rangle}(P)$ (solid dark-red triangles pointing down) of the fcc argon derived from our $C_{i j}(P)$ shown in Fig. 2(a). Our theoretical $V_{T\langle 100\rangle}(P)$ and $V_{T\langle 110\rangle}(P)$ are represented by solid (red) lines. Solid (blue) circles indicate the scattering $V_{T}$ values measured for a polycrystalline sample by Chen et al. [15]. Other previous experimental and theoretical results are shown in the same way as in Fig. 1.

diate EOS and $B(P)$ reported in Ref. [8] for the following data treatment. The three elastic constants $C_{11}(P), C_{12}(P), C_{44}(P)$ of the fcc argon were derived using the equations

$$
\begin{gathered}
\sqrt{\frac{C_{11}(P)}{\rho(P)}}=V_{L \min }(P)=V_{L\langle 100\rangle}(P), \\
C_{12}(P)=\frac{3 B(P)-C_{11}(P)}{2}, \\
C_{44}(P)=\frac{3 C^{*}(P)-C_{11}(P)-C_{12}(P)}{4},
\end{gathered}
$$

where

$$
\sqrt{\frac{C^{*}(P)}{\rho(P)}}=V_{L \max }(P)=V_{L\langle 111\rangle}(P) .
$$

Results of this treatment, namely, the experimental $C_{i j}(P)$ for the fcc argon, are summarized in Fig. 2(a). Here, we can recognize that in the earlier experimental work [14], $C_{11}(P)$ was overestimated and $C_{12}(P)$ underestimated while in the earliest theoretical work [11] both dependencies were strongly overestimated. In all other theoretical works but one, $C_{11}(P)$ was overestimated and $C_{12}(P)$ underestimated independent of the calculation approach. The calculations of Pechenik et al. [13], where a relatively simple many-body model of interatomic potentials was used, agree almost perfectly with our measurements. Interestingly, contribution of the many-body term in that theoretical work was found to be strong only for $C_{44}(P)$ while for $C_{11}(P)$ and $C_{12}(P)$ it was negligible. Values of the latter two moduli were mostly defined by the choice of the Buckingham (exp-6) pair potential whose parameters were derived by fitting the theoretical EOS to the experimental ones [13]. Another consequence of this is a good agreement of the Zener ratio derived from the $C_{i j}(P)$ predicted in Ref. [13] with our experimental values concentrated around $A=7$ [Fig. 2(b)]. Some of our experimental values are very high, around $A=16$, but the error bars are also very large in such cases. This can be explained by a small difference between $C_{11}(P)$ and $C_{12}(P)$ in the fcc argon which strongly amplifies the contribution of the experimental uncertainties to the scattering of $A=2 C_{44} /\left(C_{11}-C_{12}\right)$ as well as to the enlargement of the error bars. Nevertheless, we can conclude that our experimental $A(P)$ is much larger than those reported in the earlier experimental and theoretical works except Ref. [13] [Fig. 2(b)]. Another possible explanation, at least partial, of the low $V_{L \min }(P)$ values and, accordingly, of the small difference between $C_{11}(P)$ and $C_{12}(P)$ in our measurements could be the presence of a softer material, for example, in the form of disordered regions around the grain boundaries of the crystalline argon. However, a detailed examination of this possibility is not a subject of the present paper. In Fig. 3, we compare our experimental Cauchy discrepancy $\left(C_{12}-C_{44}-\right.$ $2 P$ ) which is negative but whose magnitude grows slower with compression than in any earlier experimental or theoretical work. The closest theoretical result is, obviously, that derived from the $C_{i j}(P)$ calculated in Ref. [13]. The small Cauchy discrepancy implies a weaker noncentral interactions in the fcc argon than could be expected from the earlier experiments and theoretical predictions, including ours.

From our experimental $C_{i j}(P)$, we derived shear modulus $G_{H}(P)$ for a polycrystalline texture-free sample of fcc argon applying the Voigt-Reuss-Hill approximation [Fig. 7(a)] and found an unexpectedly good agreement with the $G(P)$ obtained directly from the averaged transversal sound velocities $V_{T \text { av }}(P)$ measured by Marquardt et al. using the classical BLS [16]. This agreement mutually confirms reliability of the two experimental data sets and supports the earlier finding of Marquardt et al. that shear strength $\tau$ of the fcc argon at high pressures is very low [16]. To do this, Marquardt et al. used their $G(P)$ [Fig. 7(a)] and the earlier measured by Mao et al. [17], average differential strain $\langle Q\rangle=t /(6 G)$ in solid argon compressed in a DAC to similar pressures. The value of $\langle Q\rangle$ was obtained from XRD measurements of interplanar distances $d(h k l)$ of argon (corresponding to 
different directions in a single crystal) whose relative changes on compression in a DAC are different due to the presence of a uniaxial stress component $t=2 \tau$. At $P \simeq 65 \mathrm{GPa}$, the shear strength of solid argon was estimated by Marquardt et al. [16] to be $\tau \simeq 0.8 \mathrm{GPa}$ which is significantly lower than the value of $\tau \simeq 1.8 \mathrm{GPa}$ previously estimated by Mao et al. [17]. If we take into account scattering of the shear modulus in the work of Marquardt et al. [16] with the amplitude of $\pm 10 \mathrm{GPa}$, the shear strength of solid argon at $P \simeq 65 \mathrm{GPa}$ will remain below $0.92 \mathrm{GPa}$ and thus similar to that of solid neon at the same pressure (see Fig. 5 in Ref. [16]). Accordingly, at pressures below $70 \mathrm{GPa}$ both solidified noble gases are similarly soft and similarly suitable as pressure transmitting media providing quasihydrostatic load conditions. Such estimations of $\tau$ could also be done using our $G_{H}(P)$ but the resulting values of $\tau$ will only scatter more strongly [proportional to the stronger scattering of our $G_{H}(P)$ caused by the accumulation of uncertainties in our $\left.C_{i j}(P)\right]$ when compared with those obtained using Ref. [16].

The overestimated $\tau(P)$ of solid argon reported by Mao et al. [17] can be explained by the application of the overestimated shear modulus $G_{H}(P)$ [Fig. 7(a)] from the classical BLS data reported in the pioneering work of Grimsditch et al. [11] (see Introduction for more details). Moreover, since the experimental $C_{i j}(P)$ in Ref. [11] were measured up to $30 \mathrm{GPa}$ only [Fig. 7(a)], Mao et al. appear to have linearly extrapolated them to higher pressures, thus further overestimating $\tau$ at pressures around $65 \mathrm{GPa}$. Use of such a simple extrapolation is not surprising because no theoretically supported analytical equation describing the dependence of the shear modulus on pressure is available, in contrast to multiple versions of equation of state $\rho(P)$.

For completeness, we derived pressure dependencies of the fastest and the slowest transversal sound velocities of the fcc argon, $V_{T\langle 100\rangle}(P)$ and $V_{T\langle 110\rangle}(P)$, respectively, using our experimental $C_{i j}(P)$ and compared with the previous classical BLS measurements and with theoretical predictions [Fig. 7(b)]. As above for $V_{L}$ (Fig. 1), our experimental $V_{T\langle 100\rangle}(P)$ and $V_{T\langle 110\rangle}(P)$ differ more strongly from each other than in the earlier classical BLS experiments [14]. The observation that the $V_{T \text { av }}$ data points reported by Chen et al. [15] approach the slowest transversal sound velocity in a single crystal of cubic argon, namely $V_{T\langle 110\rangle}(P)$, can be of the same origin as in the case of the longitudinal sound velocities. While our experimental $V_{T\langle 100\rangle}(P)$ agrees with all other experiments and calculations, our experimental $V_{T\langle 110\rangle}(P)$ agrees only with that predicted by Pechenik et al. [13] [Fig. 7(b)]. In all other theoretical approaches and measurements, $V_{T\langle 110\rangle}(P)$ was significantly overestimated.

An alternative explanation of a stronger deviation of the experimental $n \cdot V_{L \max }(P)$ from $n \cdot V_{L \min }(P)$ than predicted in the ab initio calculations could be the coexistence of the fcc argon with another phase in a broad pressure range. Errandonea et al. concluded from their synchrotron-based XRD measurements that above $\sim 49 \mathrm{GPa}$ the hcp phase appears and coexists with the fcc phase up to very high pressures approaching $300 \mathrm{GPa}$ [2]. Because our TDBS technique exhibits a high spatial resolution, even small inclusions of the hcp phase could be detected in our samples, provided elastic anisotropy of the hep phase is much stronger or the longitudinal sound velocities

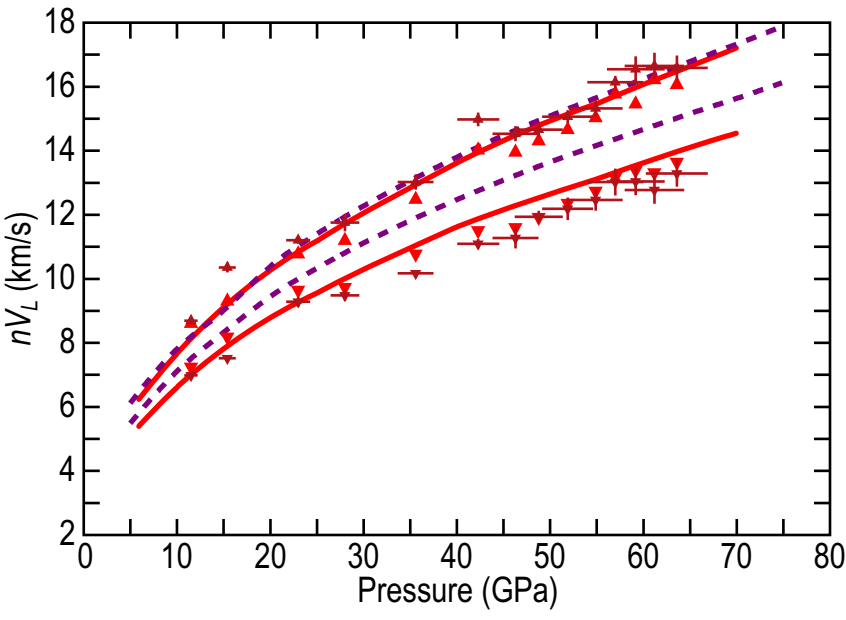

FIG. 8. Comparison of the maximal and minimal products $n \cdot V_{L}$ for the fcc phase (solid red lines) and hcp phase (dashed violet lines) of solid argon at high pressures calculated in this paper. The theoretical results are compared with our experimental $n(P) \cdot V_{L \max }(P)$ and $n(P) \cdot V_{L \min }(P)$ obtained for the two temporal windows applied to the TDBS signals. Meaning of all symbols is the same as in Fig. 1.

differ strongly from those of the fcc phase. To examine this possibility, we performed DFT calculations of $C_{i j}(P)$ and $n(P)$ of the hcp argon using the structural information reported in Ref. [2] as the input. Results of these calculations, shown in Fig. 8, disproved the possibility to detect the hcp phase in our TDBS measurements: the highest value of the product $n \cdot V_{L}$, corresponding to the crystallographic direction $\langle 001\rangle$ in the hcp structure, is identical to the maximal value in the fcc phase, $n(P) \cdot V_{L\langle 111\rangle}(P)$. The lowest value of the product $n \cdot V_{L}$ does not correspond in the hcp phase to a specific crystallographic direction and was found at each pressure from the calculated dependence of $V_{L}$ on the angle between the sound propagation direction and the $c$ axis of the hcp crystal. Within the pressure region of our work, the product $n \cdot V_{L \min }$ for the hcp phase of argon was calculated to be significantly larger than that of the fcc phase (Fig. 8). Thus, even in the case of the presence of the hcp phase in our sample, we would not be able to recognize it using the present version of the TDBS setup.

\section{CONCLUSION}

In this paper, we have measured the maximal and minimal values of the product $n \cdot V_{L}$ for single crystals of solid argon compressed up to $64 \mathrm{GPa}$. At this pressure, the density of solid argon is more than two times higher than that just after its solidification at $P=1.2 \mathrm{GPa}$ [8]. We have shown theoretically that the hcp phase of argon, potentially coexisting with the main fcc phase at high pressures, could not contribute to the herein-measured strong deviation of the maximal and minimal $n \cdot V_{L}$ values. Accordingly, applying the envelope method and our theoretical $n(P)$ for the fcc phase, we determined, with a high degree of confidence, pressure dependencies of the fastest and slowest longitudinal sound velocities in a single crystal of the fcc argon, $V_{L\langle 111\rangle}(P)$ and $V_{L\langle 100\rangle}(P)$, respectively. These values are reliable because 
we used the TDBS technique adapted to examination of transparent samples with a unique sub- $\mu \mathrm{m}$ axial resolution combined with a micrometric lateral resolution. This permits collection of high-resolution continuous 3D distributions of elastic inhomogeneities (proportional to the product $n \cdot V_{L}$ ) in microscopic transparent samples compressed in a DAC to megabar pressures. It is important to emphasize here that the spatial resolution of the TDBS technique does not degrade with pressure. Using the earlier measured EOS, we derived $C_{i j}(P), G_{H}(P)$ and estimated the transversal sound velocities $V_{T\langle 100\rangle}(P)$ and $V_{T\langle 110\rangle}(P)$ of the fcc argon up to $64 \mathrm{GPa}$. Our $G_{H}(P)$ was found to closely match $G(P)$ derived directly from the averaged transversal sound velocities $V_{T \text { av }}(P)$ measured using the classical BLS technique. This confirms the earlier finding that the shear strength of solid argon is similar to that of solid neon and, accordingly, that these two solidified noble gases are equally suitable as pressure transmitting media. The theoretical many-body model based on the Buckingham (exp-6) pair potential [13] predicted $C_{i j}(P)$ of the fcc argon, agreeing well with our experimental results. All other theoretical models, including the $a b$ initio approach used here, could not predict the measured minimum sound velocities, here $V_{L\langle 100\rangle}(P)$ and $V_{T\langle 110\rangle}(P)$. As in the case of cubic $\mathrm{H}_{2} \mathrm{O}$ ice, a strong elastic anisotropy of solid argon was revealed in this work. However, the result is valid when solid argon contains the fcc and hcp phases only. The magnitude of the Cauchy discrepancy $\left(C_{12}-C_{44}-2 P\right)$ for the fcc argon was found to increase much slower with pressure than could be concluded from the earlier experimental and theoretical works. Accordingly, the pairwise interactions in solid argon also vanish slowly upon compression.

\section{ACKNOWLEDGMENTS}

The work was supported by grants of the French National Research Agency (ANR): ANR-11-BS09-LUDACism and ANR-18-CE42-I2T2M. Technical support of R. Boehler is acknowledged.
[1] D. A. Young, Phase Diagrams of the Elements (University of California Press, Berkeley, CA, 1991).

[2] D. Errandonea, R. Boehler, S. Japel, M. Mezouar, and L. R. Benedetti, Structural transformation of compressed solid Ar: An x-ray diffraction study to 114 GPa, Phys. Rev. B 73, 092106 (2006).

[3] I. Kwon, L. A. Collins, J. D. Kress, and N. Troullier, Firstprinciples study of solid Ar and $\mathrm{Kr}$ under high compression, Phys. Rev. B 52, 15165 (1995).

[4] A. K. McMahan, Structural transitions and metallization in compressed solid argon, Phys. Rev. B 33, 5344 (1986).

[5] P. Schwerdtfeger, N. Gaston, R. P. Krawczyk, R. Tonner, and G. E. Moyano, Extension of the Lennard-Jones potential: Theoretical investigations into rare-gas clusters and crystal lattices of $\mathrm{He}, \mathrm{Ne}, \mathrm{Ar}$, and $\mathrm{Kr}$ using many-body interaction expansions, Phys. Rev. B 73, 064112 (2006).

[6] L. W. Finger, R. M. Hazen, G. Zou, H. K. Mao, and P. M. Bell, Structure and compression of crystalline argon and neon at high pressure and room temperature, Appl. Phys. Lett. 39, 892 (1981).

[7] D. A. Dzivenko, A. Zerr, R. Boehler, and R. Riedel, Equation of state of cubic hafnium(IV) nitride having $\mathrm{Th}_{3} \mathrm{P}_{4}$-type structure, Solid State Commun. 139, 255 (2006).

[8] P. Richet, J.-A. Xu, and H.-K. Mao, Quasi-hydrostatic compression of ruby to 500 Kbar, Phys. Chem. Miner. 16, 207 (1988).

[9] A. Zerr, H. Reichmann, H. Euler, and R. Boehler, Hydrostatic compression of $\gamma-\left(\mathrm{Mg}_{0.6}, \mathrm{Fe}_{0.4}\right)_{2} \mathrm{SiO}_{4}$ to $50.0 \mathrm{GPa}$, Phys. Chem. Miner. 19, 507 (1993).

[10] M. Aoki and T. Kurokawa, A simple environment-dependent overlap potential and Cauchy violation in solid argon, J. Phys.: Condens. Matter 19, 236228 (2007).

[11] M. Grimsditch, P. Loubeyre, and A. Polian, Brillouin scattering and three-body forces in argon at high pressures, Phys. Rev. B 33, 7192 (1986).

[12] T. Iitaka and T. Ebisuzaki, First-principles calculation of elastic properties of solid argon at high pressures, Phys. Rev. B 65 , 012103 (2001).
[13] E. Pechenik, I. Kelson, and G. Makov, Many-body model of rare gases at high pressures, Phys. Rev. B 78, 134109 (2008).

[14] H. Shimizu, H. Tashiro, T. Kume, and S. Sasaki, High-Pressure Elastic Properties of Solid Argon to 70 GPa, Phys. Rev. Lett. 86, 4568 (2001).

[15] B. Chen, A. E. Gleason, J. Y. Yan, K. J. Koski, S. Clark, and R. Jeanloz, Elasticity, strength, and refractive index of argon at high pressures, Phys. Rev. B 81, 144110 (2010).

[16] H. Marquardt, S. Speziale, A. Gleason, S. Sinogeikin, I. Kantor, and V. B. Prakapenka, Brillouin scattering and x-ray diffraction of solid argon to $65 \mathrm{GPa}$ and $700 \mathrm{~K}$ : Shear strength of argon at HP/HT, J. Appl. Phys. 114, 093517 (2013).

[17] H.-k. Mao, J. Badro, J. Shu, R. J. Hemley, and A. K. Singh, Strength, anisotropy, and preferred orientation of solid argon at high pressures, J. Phys.: Condens. Matter 18, S963 (2006).

[18] M. Kuriakose, S. Raetz, Q. M. Hu, S. M. Nikitin, N. Chigarev, V. Tournat, A. Bulou, A. Lomonosov, P. Djemia, V. E. Gusev, and A. Zerr, Longitudinal sound velocities, elastic anisotropy, and phase transition of high-pressure cubic $\mathrm{H}_{2} \mathrm{O}$ ice to $82 \mathrm{GPa}$, Phys. Rev. B 96, 134122 (2017).

[19] V. E. Gusev and P. Ruello, Advances in applications of timedomain Brillouin scattering for nanoscale imaging, Appl. Phys. Rev. 5, 031101 (2018).

[20] M. Kuriakose, S. Raetz, N. Chigarev, S. M. Nikitin, A. Bulou, D. Gasteau, V. Tournat, B. Castagnede, A. Zerr, and V. E. Gusev, Picosecond laser ultrasonics for imaging of transparent polycrystalline materials compressed to megabar pressures, Ultrasonics 69, 259 (2016).

[21] S. M. Nikitin, N. Chigarev, V. Tournat, A. Bulou, D. Gasteau, B. Castagnede, A. Zerr, and V. E. Gusev, Revealing sub- $\mu \mathrm{m}$ and $\mu$ m-scale textures in $\mathrm{H}_{2} \mathrm{O}$ ice at megabar pressures by timedomain Brillouin scattering, Sci. Rep. 5, 09532 (2015).

[22] M. Grimsditch, R. Letoullec, A. Polian, and M. Gauthier, Refractive index determination in diamond anvil cells: Results for argon, J. Appl. Phys. 60, 3479 (1986).

[23] A. Chopelas and R. Boehler, $\mathrm{MgO}: \mathrm{V}^{2+}$ pressure scale for diamond anvil cells, Mater. Res. Soc. Symp. Proc. 22, 275 (1984). 
[24] H. K. Mao, J. Xu, and P. M. Bell, Calibration of the ruby pressure gauge to $800 \mathrm{kbar}$ under quasi-hydrostatic conditions, J. Geophys. Res.-Sol. Ea. 91, 4673 (1986).

[25] G. Kresse and J. Furthmüller, Efficiency of ab-initio total energy calculations for metals and semiconductors using a plane-wave basis set, Comput. Mater. Sci. 6, 15 (1996).

[26] G. Kresse and J. Hafner, Ab initio molecular dynamics for liquid metals, Phys. Rev. B 47, 558 (1993).

[27] G. Kresse and J. Furthmüller, Efficient iterative schemes for ab initio total-energy calculations using a plane-wave basis set, Phys. Rev. B 54, 11169 (1996).

[28] MedeA-VASP, VASP license LSPM-CNRS-Université Paris 13.

[29] MedeA, Materials Exploration and Design Analysis (Materials Design Inc.), http://www.materialsdesign.com (1998-2019).

[30] Y.-S. Kim, K. Hummer, and G. Kresse, Accurate band structures and effective masses for InP, InAs, and InSb using hybrid functionals, Phys. Rev. B 80, 035203 (2009).

[31] A. V. Krukau, O. A. Vydrov, A. F. Izmaylov, and G. E. Scuseria, Influence of the exchange screening parameter on the performance of screened hybrid functionals, J. Chem. Phys. 125, 224106 (2006).

[32] J. Paier, M. Marsman, K. Hummer, G. Kresse, I. C. Gerber, and J. G. Ángyán, Screened hybrid density functionals applied to solids, J. Chem. Phys. 124, 154709 (2006).

[33] J. Paier, M. Marsman, K. Hummer, G. Kresse, I. C. Gerber, and J. G. Ángyán, Erratum: Screened hybrid density functionals applied to solids [J. Chem. Phys. 124, 154709 (2006)], J. Chem. Phys. 125, 249901 (2006).

[34] J. Heyd, J. E. Peralta, G. E. Scuseria, and R. L. Martin, Energy band gaps and lattice parameters evaluated with the HeydScuseria-Ernzerhof screened hybrid functional, J. Chem. Phys. 123, 174101 (2005).

[35] G. Kresse and D. Joubert, From ultrasoft pseudopotentials to the projector augmented-wave method, Phys. Rev. B 59, 1758 (1999).

[36] H. J. Monkhorst and J. D. Pack, Special points for Brillouinzone integrations, Phys. Rev. B 13, 5188 (1976).

[37] M. Gajdoš, K. Hummer, G. Kresse, J. Furthmüller, and F. Bechstedt, Linear optical properties in the projector-augmented wave methodology, Phys. Rev. B 73, 045112 (2006). 